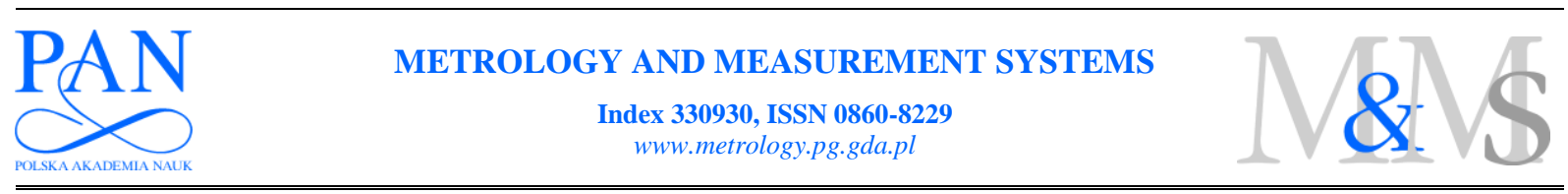

\title{
SYSTEMATIC ERRORS OF THE LIDFT METHOD: ANALYTICAL FORM AND VERIFICATION BY A MONTE CARLO METHOD
}

\author{
Józef Borkowski \\ Wroclaw University of Technology, Chair of Electronic and Photonic Metrology, Bolestawa Prusa 53/55, 50-317 Wroctaw, Poland \\ ( $₫$ Jozef.Borkowski@pwr.wroc.pl, +48 71320 6329)
}

\begin{abstract}
This paper derives analytical formulas for the systematic errors of the linear interpolated DFT (LIDFT) method when used to estimating multifrequency signal parameters and verifies this analysis using Monte-Carlo simulations. The analysis is performed on the version of the LIDFT method based on optimal approximation of the unit circle by a polygon using a pair of windows. The analytical formulas derived here take the systematic errors in the estimation of amplitude and frequency of component oscillations in the multifrequency signal as the sum of basic errors and the errors caused by each of the component oscillations. Additional formulas are also included to analyze particular quantities such as a signal consisting of two complex oscillations, and the analyses are verified using Monte-Carlo simulations.
\end{abstract}

Keywords: LIDFT, multifrequency signal, interpolated DFT, spectrum estimation, zero padding, unit circle, approximation by polygon.

\section{Introduction}

Modern measurement techniques use sophisticated methods based on digital signal processing (DSP) that have become increasingly sophisticated to handle more complex mathematical problems [1-6]. An important class of complex problems is spectrum estimation, understood as the estimation of multifrequency signal parameters. The most accurate methods of this type of estimation are the Prony method and its modifications, the transmittance modeling methods and subspace methods (based mainly on the properties of the signal autocorrelation matrix and its eigenvalues) [1-4, 7-8]. The interpolated spectrum methods yield less accurate results but require a significantly shorter computation time. This class of methods includes nonparametric spectrum interpolation methods [9-16] and interpolated methods based on DFT [17-44]. A special class is non-iterative methods that take the leakage of the spectrum into account in their equations, including Multipoint Weighted Interpolated DFT (MWIDFT) methods [32, 34, 37] and the Linear Interpolated DFT (LIDFT) method [29-31, 41-42, 45-46]. The group of MWIDFT methods is only defined for the maximum sidelobe decay windows (class I Rife-Vincent windows) [17, 34, 37, 39]. The most advanced MWIDFT method appears to be the one based on complex spectrum values that approximate the spectrum leakage with a polynomial and a step 1 bin [39]. However, the MWIDFT methods have limitations, mainly with respect to frequency resolution and the level of oscillation amplitude versus the level of spectrum leakage. The LIDFT method can be used where former interpolation methods have failed by considering the spectrum leakage. The LIDFT method has evolved, and the latest version [42] is based on optimal approximation of the unit circle by a polygon [42, 45-46] and the use of a pair of windows [42]. The current 
paper addresses the systematic errors of this latest version of the LIDFT method.

\section{Unit circle approximation and the pair of windows in the LIDFT method}

Let us assume that the aim of estimation is to determine the parameters $A_{k}, \omega_{k}=2 \pi f_{k}$, and $\varphi_{k}$ of the following multifrequency signal, defined in the domain of time $t$ :

$$
y(t)=\sum_{k \in S} A_{k} \sin \left(\omega_{k} t+\varphi_{k}\right), \quad S=\left\{k_{1}, \ldots, k_{K}\right\},
$$

where the assumption $k=k_{1}, \ldots, k_{K}$ (in which the condition $k_{i+1}=k_{i}+1$ is not required) instead of $k=1, \ldots, K$ allows for the integer index $k$ to be linked with the appropriate integer index of the DFT spectrum sample. This assumption involves a simple renumbering of components that simplifies the description of the LIDFT method by coding additional information about the rough location of components in the DFT spectrum within the index $k$.

Equation (1) can be written by:

$$
y(t)=\sum_{k \in S_{1}} B_{k} e^{j \omega_{k} t}, \quad S_{1}=\left\{k_{1}, \ldots, k_{P}\right\},
$$

where: $B_{k}=\left(A_{k} /(2 j)\right) e^{j \varphi_{k}}, P=2 K, B_{k_{P-m+1}}=B_{k_{m}}^{*}, e^{j \omega_{k P-m+1}}=\left(e^{j \omega_{k_{m}}}\right)^{*}, m=1, \ldots, K$.

Signal (2) is sampled with a frequency $f_{\mathrm{s}}=1 / T\left(f_{\mathrm{s}} / 2>\max _{k}\left\{f_{k}\right\}\right.$, where $\left.f_{k}=\omega_{k} /(2 \pi)\right)$ results in $N$ samples (indexed in general by $n=n_{0}, \ldots, n_{0}+N-1$ from the starting point $n_{0}$ ) and has the form:

$$
y_{n}=y(t=n T)=\sum_{k \in S_{1}} B_{k} e^{j 2 \pi f_{k} n T}=\sum_{k \in S_{1}} B_{k} e^{j 2 \pi n \lambda_{k} / N}, \quad S_{1}=\left\{k_{1}, \ldots, k_{P}\right\},
$$

where $\lambda_{k}=N f_{k} / f_{\mathrm{s}}$ is the normalized frequency in the DFT bin and $B_{k}$ is the complex amplitude of the $k$-th complex oscillation $B_{k} e^{j 2 \pi n \lambda_{k} / N}$. The starting point $n_{0}$ for the sample index $n$ theoretically does not change the frequency and amplitude estimation results when the spectrum leakage is negligible but only changes the phase (which can be easy recalculated for different values of $n_{0}$ ). Most often $n_{0}=0$, but sometimes it is useful to assume that $n_{0}=-N / 2$ when $N$ is even to make the time axis symmetrical, simplifying many equations and algorithm derivations.

The LIDFT method presented in [42] makes two assumptions:

1) Approximation of the unit circle by a polygon (with the parameter $R \geq 1$ that allows the number of sides of the approximation polygon to be varied) [45, 50, 51], defined by:

$$
\begin{gathered}
e^{-j 2 \pi n \lambda_{k} / N} \approx e^{-j 2 \pi n k / M}\left[\alpha_{n}+j \gamma_{k} \beta_{n}\right], \quad M=N R, \\
\lambda_{k}=\frac{1}{R}\left(k+\gamma_{k}\right), \quad \gamma_{k} \in[-1 / 2,1 / 2],
\end{gathered}
$$

where $k \in S_{1}$ (here, the indexing used in (1) is very useful) and the parameter $R$ is selected such that $M$ is an integer (usually as a power of 2 when the radix-2 FFT algorithm is used in the final estimation method). $\alpha_{n}$ and $\beta_{n}$ are defined with parameters $\eta_{1}$ and $\eta_{2}$, respectively:

$$
\alpha_{n}\left(\eta_{1}\right)=\left(1-\eta_{1}\right) \cos x_{n}+\eta_{1}, \quad \beta_{n}\left(\eta_{2}\right)=-2 \cdot\left[\left(1-\eta_{2}\right) \sin x_{n}+\eta_{2} \tan x_{n}\right],
$$

where:

$$
n=-N / 2, \ldots, N / 2-1, \quad x_{n}=\pi n / M
$$

and optimal parameters values $\eta_{1}$ and $\eta_{2}$ that minimize maximum approximation errors should be found in the triangle on the plane $\left(\eta_{1}, \eta_{2}\right)$ given by the following conditions $[42,45,46]$ : 


$$
1 / 4 \leq \eta_{1} \leq 1 / 2, \quad 1 / 12 \leq \eta_{2} \leq 1 / 3, \quad \eta_{2}-\eta_{1} \leq-1 / 6 .
$$

2) The pair of windows, the even window $h_{n}$ and the odd window $g_{n}$, are obtained from the prototype window $w_{n}$ by:

$$
h_{n}=w_{n}^{2} \alpha_{n}, \quad g_{n}=w_{n}^{2} \beta_{n} .
$$

Simulations performed with $w_{n}$ as a triangular window [42, 45, 46] show that parameter values $\eta_{1} \approx 1 / 2$ and $\eta_{2} \approx 1 / 6$ are close to optimal for this window, but when other windows are used, these values should be verified as being close to optimal.

After applying the above two assumptions (unit circle approximation and the pair of windows) in DFT formulas and denoting $C_{k}=\gamma_{k} B_{k}$ with $\gamma_{k}=R \lambda_{k}-k$ obtained from (5), the linear matrix equation is derived [42], which has the form $\mathbf{F x}=\mathbf{f}$ :

$$
\left[\begin{array}{cc}
r_{i-k} & s_{i-k} \\
p_{i-k} & q_{i-k}
\end{array}\right]_{2 P \times 2 P}\left[\begin{array}{l}
B_{k} \\
C_{k}
\end{array}\right]_{2 P \times 1}=\left[\begin{array}{l}
u_{i} \\
v_{i}
\end{array}\right]_{2 P \times 1}, \quad i, k \in S_{1},
$$

where $S_{1}$ is the set of initial locations of components in the spectrum, i.e., (5) implies the condition for each integer $k \in S_{1}$ :

$$
\lambda_{k} \in[(k-0.5) / R,(k+0.5) / R] .
$$

The elements of the matrices in (10) are given by:

$$
\begin{gathered}
r_{m}=\mathrm{FFT}_{m}\left\{\alpha_{n} h_{n}\right\}_{N R}, \quad s_{m}=-j \mathrm{FFT}_{m}\left\{\beta_{n} h_{n}\right\}_{N R}, \quad p_{m}=j \mathrm{FFT}_{m}\left\{\alpha_{n} g_{n}\right\}_{N R}, \\
q_{m}=\mathrm{FFT}_{m}\left\{\beta_{n} g_{n}\right\}_{N R}, \quad u_{m}=\mathrm{FFT}_{m}\left\{y_{n} h_{n}\right\}_{N R}, \quad v_{m}=j \mathrm{FFT}_{m}\left\{y_{n} g_{n}\right\}_{N R}, \\
m=0, \ldots, N R-1,
\end{gathered}
$$

where $\operatorname{FFT}_{m}\left\{z_{n}\right\}_{N R}$ denotes the $m$-th element of the set of values obtained by the FFT algorithm applied to the set of $N$ values $z_{n}$ supplemented with zeros to form the $N R$ element set as follows:

$$
\left\{z_{n}\right\}_{N R}=\left\{z_{0}, \ldots, z_{N / 2-1}, 0, \ldots, 0, z_{-N / 2}, \ldots, z_{-1}\right\}_{N R} .
$$

The solution of (10) allows for the direct determination of the amplitudes $B_{k}$ and the normalized frequencies $\lambda_{k}$ from:

$$
\lambda_{k}=\frac{k+\operatorname{Re} \gamma_{k}}{R}, \quad \gamma_{k}=C_{k} / B_{k},
$$

where $\operatorname{Re} \gamma_{k}$ is introduced instead of $\gamma_{k}$ because the result is usually not real but is complex due to the presence of noise in the signal, the finite accuracy of calculations and the possible nonideal initial location of the components of the spectrum. When this initial location is properly defined, then $\left|\operatorname{Re} \gamma_{k}\right| \leq 1 / 2$, and if it is not properly defined, several iterations can be performed [41]. The values $\operatorname{Im} \gamma_{k}$, which should be close to 0 , can also be used to verify that the initial locations of the components are correct.

\section{Systematic errors of the LIDFT method for the case of a multifrequency signal}

For the signal consisting of $P$ complex oscillations (each with a complex amplitude $B_{k}$ and a normalized frequency $\lambda_{k}$ ) and with condition (11), the signal samples $y_{n}$ are defined by (3), and applying (10) and (12)-(14) allows us to obtain the estimators $\hat{B}_{k}$ and $\hat{C}_{k}$ instead of the exact values $B_{k}$ and $C_{k}=B_{k} \gamma_{k}$ due to the systematic errors of the LIDFT method. (10), taking (3) into account, takes on the form: 


$$
\left[\begin{array}{cc}
r_{i-k} & s_{i-k} \\
p_{i-k} & q_{i-k}
\end{array}\right]_{2 P \times 2 P}\left[\begin{array}{l}
\hat{B}_{k} \\
\hat{C}_{k}
\end{array}\right]_{2 P \times 1}=\left[\begin{array}{c}
H_{i-k}\left(-\gamma_{k}\right) \\
G_{i-k}\left(-\gamma_{k}\right)
\end{array}\right]_{2 P \times P}\left[B_{k}\right]_{P \times 1}, \quad i, k \in S_{1},
$$

in which $H_{i-k}\left(-\gamma_{k}\right)$ and $G_{i-k}\left(-\gamma_{k}\right)$ are defined by:

$$
H_{m}(\gamma)=H\left(\frac{m+\gamma}{R}\right), \quad G_{m}(\gamma)=G\left(\frac{m+\gamma}{R}\right), \quad|\gamma| \leq 1 / 2,
$$

where $m=i-k$ is the integer number, $\gamma=-\gamma_{k}$, functions $H(\lambda)$ and $G(\lambda)$ are defined for any real $\lambda$, are periodic with period $N($ e.g., $H(\lambda+N)=H(\lambda))$ and are shifted Discrete-time Fourier Transforms (DtFT) of $N$ samples of windows $h_{n}$ and $j g_{n}$ :

$$
H(\lambda)=\sum_{n=-N / 2}^{N / 2-1} h_{n} e^{-j 2 \pi n \lambda / N}, \quad G(\lambda)=j \sum_{n=-N / 2}^{N / 2-1} g_{n} e^{-j 2 \pi n \lambda / N} .
$$

By introducing $M_{m k}$ and $N_{m k}$ according to:

$$
\left[\begin{array}{cc}
r_{i-m} & s_{i-m} \\
p_{i-m} & q_{i-m}
\end{array}\right]_{2 P \times 2 P}\left[\begin{array}{c}
M_{m k} \\
N_{m k}
\end{array}\right]_{2 P \times P}=\left[\begin{array}{c}
H_{i-k}\left(-\gamma_{k}\right) \\
G_{i-k}\left(-\gamma_{k}\right)
\end{array}\right]_{2 P \times P}, \quad i, m, k \in S_{1},
$$

based on (17) and (20):

$$
\hat{B}_{k}=\sum_{i \in S_{1}} M_{k i} B_{i}, \quad \hat{C}_{k}=\sum_{i \in S_{1}} N_{k i} B_{i}
$$

For $\hat{B}_{k}$, the maximum (for the worst-case phase $\arg B_{i}$ ) of the systematic error $\delta_{\mathrm{d}}\left|B_{k}\right|$ of $\left|B_{k}\right|$ is derived from (21) (App. A):

$$
\delta_{\mathrm{d}}\left|B_{k}\right|=\delta_{\mathrm{d} k}\left|B_{k}\right|+\sum_{i \in S_{1}, i \neq k} \delta_{\mathrm{d} i}\left|B_{k}\right|,
$$

where:

$$
\begin{gathered}
\delta_{\mathrm{d} k}\left|B_{k}\right|=\left|M_{k k}-1\right|, \\
\delta_{\mathrm{d} i}\left|B_{k}\right|=\frac{\left|B_{i}\right|}{\left|B_{k}\right|} \cdot \delta_{\mathrm{d} i}^{\prime}\left|B_{k}\right|, \quad \delta_{\mathrm{d} i}^{\prime}\left|B_{k}\right|=\left|M_{k i}\right|
\end{gathered}
$$

are the components of total error (22).

Analogously to (22)-(24), the maximum (for the worst-case phase $\arg B_{i}$ ) of the systematic error $\Delta_{\mathrm{d}} \lambda_{k}$ of $\lambda_{k}$ is derived (App. B):

$$
\Delta_{\mathrm{d}} \lambda_{k}=\Delta_{\mathrm{d} k} \lambda_{k}+\sum_{i \in S_{1}, i \neq k} \Delta_{\mathrm{d} i} \lambda_{k},
$$

where:

$$
\begin{gathered}
\Delta_{\mathrm{d} k} \lambda_{k}=R^{-1}\left|\left(N_{k k}-\gamma_{k}\right)-\gamma_{k}\left(M_{k k}-1\right)\right|, \\
\Delta_{\mathrm{d} i} \lambda_{k}=\frac{\left|B_{i}\right|}{\left|B_{k}\right|} \cdot \Delta_{\mathrm{d} i}^{\prime} \lambda_{k}, \quad \Delta_{\mathrm{d} i}^{\prime} \lambda_{k}=R^{-1}\left|N_{k i}-\gamma_{k} M_{k i}\right|
\end{gathered}
$$

are the components of total error (25).

To determine (22)-(27), $N_{m k}$ and $M_{m k}$ must be known from (20). The right side of this equation, taking (12)-(13) and (18)-(19) into account, has the form: 


$$
\begin{gathered}
{\left[\begin{array}{c}
H_{i-k}\left(-\gamma_{k}\right) \\
G_{i-k}\left(-\gamma_{k}\right)
\end{array}\right]_{2 P \times P}=\left[\begin{array}{cc}
r_{i-k} & s_{i-k} \\
p_{i-k} & q_{i-k}
\end{array}\right]_{2 P \times 2 P}\left[\begin{array}{c}
\mathbf{I} \\
\boldsymbol{\Lambda}_{\gamma}
\end{array}\right]_{2 P \times P}-\left[\begin{array}{cc}
\Delta r_{i-k}\left(\gamma_{k}\right) & \Delta s_{i-k}\left(\gamma_{k}\right) \\
\Delta p_{i-k}\left(\gamma_{k}\right) & \Delta q_{i-k}\left(\gamma_{k}\right)
\end{array}\right]_{2 P \times 2 P}\left[\begin{array}{c}
\mathbf{I} \\
\mathbf{\Lambda}_{\gamma}
\end{array}\right]_{2 P \times P},} \\
i, k \in S_{1},
\end{gathered}
$$

where $\mathbf{I}$ is an identity matrix, $\boldsymbol{\Lambda}_{\gamma}=\left[\gamma_{k}\right]$ is the diagonal matrix with elements $\gamma_{k}\left(k \in S_{1}\right)$, and using notation from (12)-(13) and $m=i-k$, the new symbols introduced in (28) are defined as:

$$
\begin{gathered}
\Delta r_{m}\left(\gamma_{k}\right)=\operatorname{FFT}_{m}\left\{\Delta \alpha_{n}\left(\gamma_{k}\right) h_{n}\right\}_{N R}, \quad \Delta s_{m}\left(\gamma_{k}\right)=-j \operatorname{FFT}_{m}\left\{\Delta \beta_{n}\left(\gamma_{k}\right) h_{n}\right\}_{N R}, \\
\Delta p_{m}\left(\gamma_{k}\right)=j \mathrm{FFT}_{m}\left\{\Delta \alpha_{n}\left(\gamma_{k}\right) g_{n}\right\}_{N R}, \quad \Delta q_{m}\left(\gamma_{k}\right)=\mathrm{FFT}_{m}\left\{\Delta \beta_{n}\left(\gamma_{k}\right) g_{n}\right\}_{N R}, \\
\Delta \alpha_{n}\left(\gamma_{k}\right)=\alpha_{n}-\cos \left(2 \gamma_{k} x_{n}\right), \quad \Delta \beta_{n}\left(\gamma_{k}\right)=\beta_{n}+\gamma_{k}^{-1} \sin \left(2 \gamma_{k} x_{n}\right) .
\end{gathered}
$$

Applying (28) to (20) yields the following result:

$$
\begin{gathered}
{\left[\begin{array}{cc}
r_{i-m} & s_{i-m} \\
p_{i-m} & q_{i-m}
\end{array}\right]_{2 P \times 2 P}\left[\begin{array}{c}
{\left[M_{m k}\right]-\mathbf{I}} \\
{\left[N_{m k}\right]-\boldsymbol{\Lambda}_{\gamma}}
\end{array}\right]_{2 P \times P}=-\left[\begin{array}{cc}
\Delta r_{i-k}\left(\gamma_{k}\right) & \Delta s_{i-k}\left(\gamma_{k}\right) \\
\Delta p_{i-k}\left(\gamma_{k}\right) & \Delta q_{i-k}\left(\gamma_{k}\right)
\end{array}\right]_{2 P \times 2 P}\left[\begin{array}{c}
\mathbf{I} \\
\boldsymbol{\Lambda}_{\gamma}
\end{array}\right]_{2 P \times P},} \\
i, m, k \in S_{1} .
\end{gathered}
$$

The result from (32), together with those from (22)-(27), form the basis for a more detailed analysis of the systematic errors of the LIDFT method for the case of two complex oscillations, presented in Section 4.

\section{The case of two complex oscillations}

Consider the case of two complex oscillations: $B_{k} e^{j 2 \pi n \lambda_{k} / N}, B_{i} e^{j 2 \pi n \lambda_{i} / N}$, where $\lambda_{i}>\lambda_{k}$, $\lambda_{k}=\left(k+\gamma_{k}\right) / R, \lambda_{i}=\left(i+\gamma_{i}\right) / R,\left|\gamma_{i}\right| \leq 1 / 2,\left|\gamma_{k}\right| \leq 1 / 2$, the numbers $i, k, \tau=i-k$ are integer values and $S_{1}=\{k, i\}$. For this case, (22)-(27) have the form:

$$
\begin{gathered}
{\left[\begin{array}{c}
\delta_{\mathrm{d}}\left|B_{k}\right| \\
\Delta_{\mathrm{d}} \lambda_{k}
\end{array}\right]=\mathbf{D} \cdot\left[\begin{array}{c}
1 \\
\left|B_{i}\right| /\left|B_{k}\right|
\end{array}\right], \quad \mathbf{D}=\left[\begin{array}{cc}
\delta_{\mathrm{d} k}\left|B_{k}\right| & \delta_{\mathrm{d} i}^{\prime}\left|B_{k}\right| \\
\Delta_{\mathrm{d} k} \lambda_{k} & \Delta_{\mathrm{d} i}^{\prime} \lambda_{k}
\end{array}\right],} \\
\mathbf{D}\left(\tau, \gamma_{k}, \gamma_{i}\right)=\operatorname{abs}\left(\left[\begin{array}{cc}
1 & 0 \\
-\gamma_{k} / R & 1 / R
\end{array}\right] \mathbf{M}\right), \quad \mathbf{M}=\left[\begin{array}{cc}
\left(M_{k k}-1\right) & M_{k i} \\
\left(N_{k k}-\gamma_{k}\right) & N_{k i}
\end{array}\right],
\end{gathered}
$$

where $\operatorname{abs}(\mathbf{X})$ is the modulus of matrix $\mathbf{X}$, i.e., for $\mathbf{X}=\left[x_{i j}\right]$, $\operatorname{abs}(\mathbf{X})=\left[\left|x_{i j}\right|\right]$.

From the assumptions $h_{n}=h_{-n}, g_{n}=-g_{-n}, h_{-N / 2}=0$ and from (32), Eqs. (C13) and (C14) are derived in App. C, implying that:

$$
2 \mathbf{M}=-\mathbf{A}^{-1}\left[\begin{array}{cc}
v-u & -v^{\prime}-u^{\prime} \\
z+x & z^{\prime}-x^{\prime}
\end{array}\right]-\mathbf{B}^{-1}\left[\begin{array}{cc}
w+u & w^{\prime}-u^{\prime} \\
y-x & -y^{\prime}-x^{\prime}
\end{array}\right],
$$

where:

$$
\mathbf{M}=\left[\begin{array}{cc}
\left(M_{k k}-1\right) & M_{k i} \\
\left(N_{k k}-\gamma_{k}\right) & N_{k i}
\end{array}\right], \quad \mathbf{A}=\left[\begin{array}{ll}
a & b \\
c & d
\end{array}\right], \quad \mathbf{B}=\left[\begin{array}{cc}
e & -b \\
-c & f
\end{array}\right] .
$$

Taking into account (12)-(13), (29)-(31), and (C6)-(C10) from App. C, the properties $h_{n}=h_{-n}, \quad g_{n}=-g_{-n}, \quad h_{-N / 2}=0 \quad$ and $\quad \alpha_{n}=\alpha_{-n}, \quad \beta_{n}=-\beta_{-n} \quad$ and considering the range $n=-N / 2, \ldots, N / 2-1$ :

$$
a=2 \sum_{n} h_{n} \alpha_{n} \sin ^{2} \tau x_{n}, \quad b=2 \sum_{n} h_{n} \beta_{n} \sin \tau x_{n} \cos \tau x_{n}, \quad c=2 \sum_{n} g_{n} \alpha_{n} \sin \tau x_{n} \cos \tau x_{n}, \text { (37) }
$$




$$
\begin{gathered}
d=2 \sum_{n} g_{n} \beta_{n} \cos ^{2} \tau x_{n}, \quad e=2 \sum_{n} h_{n} \alpha_{n} \cos ^{2} \tau x_{n}, \quad f=2 \sum_{n} g_{n} \beta_{n} \sin ^{2} \tau x_{n}, \\
u=-2 \sum_{n} h_{n}\left(\gamma_{k} \beta_{n}+\sin 2 \gamma_{k} x_{n}\right) \sin \tau x_{n} \cos \tau x_{n}, \quad v=2 \sum_{n} h_{n}\left(\alpha_{n}-\cos 2 \gamma_{k} x_{n}\right) \sin ^{2} \tau x_{n}, \\
w=2 \sum_{n} h_{n}\left(\alpha_{n}-\cos 2 \gamma_{k} x_{n}\right) \cos ^{2} \tau x_{n}, \quad x=2 \sum_{n} g_{n}\left(\alpha_{n}-\cos 2 \gamma_{k} x_{n}\right) \sin \tau x_{n} \cos \tau x_{n}, \\
y=2 \sum_{n} g_{n}\left(\gamma_{k} \beta_{n}+\sin 2 \gamma_{k} x_{n}\right) \sin ^{2} \tau x_{n}, \quad z=2 \sum_{n} g_{n}\left(\gamma_{k} \beta_{n}+\sin 2 \gamma_{k} x_{n}\right) \cos ^{2} \tau x_{n} .
\end{gathered}
$$

The relationships for $u^{\prime}, v^{\prime}, w^{\prime}, x^{\prime}, y^{\prime}$, and $z^{\prime}$ are determined by (39)-(41) after substituting $\gamma_{i}$ for $\gamma_{k}$.

The upper bounds of the component errors of matrix $\mathbf{D}$ can be obtained by:

$$
\mathbf{D}(\tau / R)=\max _{\gamma_{k}, \gamma_{i}} \operatorname{abs}\left(\left[\begin{array}{cc}
1 & 0 \\
-\gamma_{k} / R & 1 / R
\end{array}\right] \mathbf{M}\right)
$$

or by:

$$
\mathbf{D}\left(\left|\lambda_{k}-\lambda_{i}\right|\right)=\max _{\substack{\tau, \gamma_{k}, \gamma_{i}: \\
\left|\lambda_{k}-\lambda_{i}\right|=\text { const. }}} \operatorname{abs}\left(\left[\begin{array}{cc}
1 & 0 \\
-\gamma_{k} / R & 1 / R
\end{array}\right] \mathbf{M}\right)=\max _{\substack{\tau, \gamma_{k}, \gamma_{i}: \\
\tau+\gamma_{i}-\gamma_{k}=\text { const. }}} \operatorname{abs}\left(\left[\begin{array}{cc}
1 & 0 \\
-\gamma_{k} / R & 1 / R
\end{array}\right] \mathbf{M}\right) .
$$

The derivation of (42) is presented in [42]; however, the version of the equation presented here differs in the fact that the maximization with respect to $\gamma_{k}$ and $\gamma_{i}$ is only with respect to the transformation of (34) to (42), i.e., to the last step of the derivation. This also allows for a second method of maximization with respect to $\gamma_{i}$ and $\gamma_{k}$ for a given $\tau+\gamma_{I}-\gamma_{k}$, which is performed in (43). This type of maximization indicates the characteristics of the upper bounds of the component errors from matrix $\mathbf{D}$ as a function of the distance $\left|\lambda_{k}-\lambda_{i}\right|$ between the components of the spectrum. These bounds, calculated from (43) for the triangular window $w_{n}$ in (9), with $\eta_{1}=1 / 2$ and $\eta_{2}=1 / 6$ in (6), are presented in Figs. 1 and 2 and are verified using Monte-Carlo simulations in Section 5.

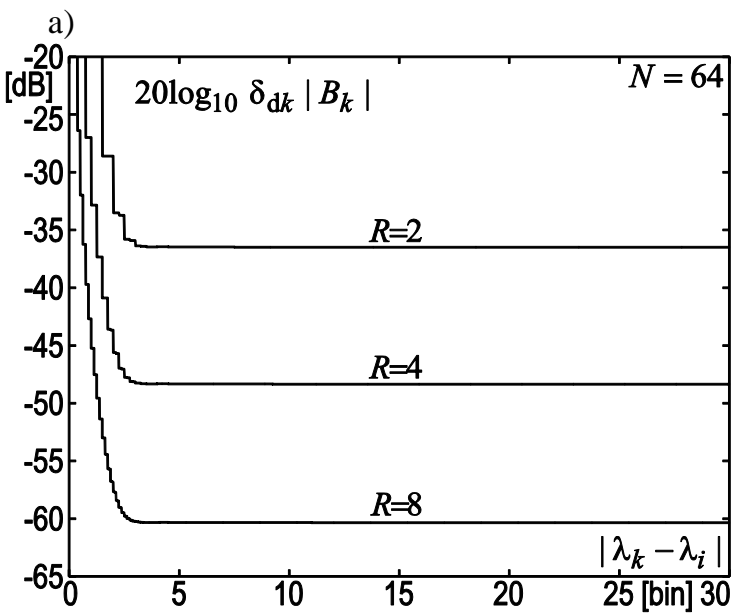

b)

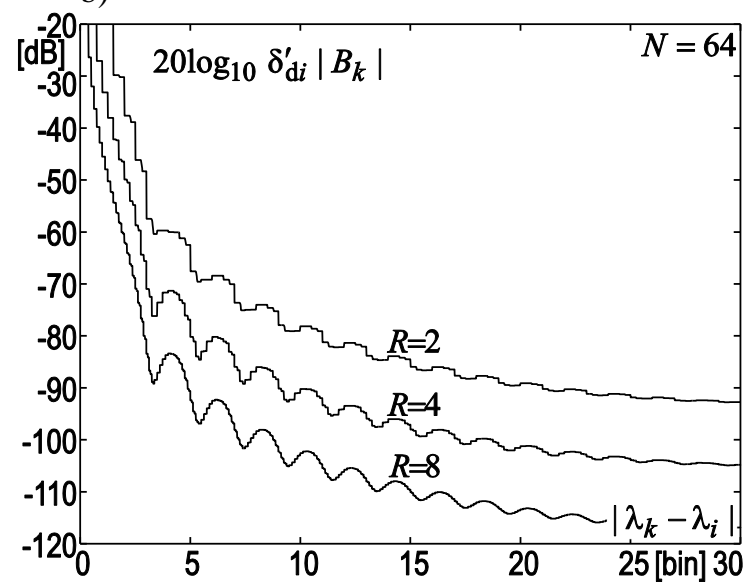

Fig. 1. Upper bounds of the component errors of the total error $\delta_{\mathrm{d}}\left|B_{k}\right|(22)$ in the estimation of $\left|B_{k}\right|$ from (33)-(41) for a signal consisting of two complex oscillations, a triangular window $w_{n}$ in (9), $\eta_{1}=1 / 2$ and $\eta_{2}=1 / 6$ : a) $\delta_{\mathrm{d} k}\left|B_{k}\right|$, b) $\delta_{\mathrm{d} i}^{\prime}\left|B_{k}\right|$. 
a)

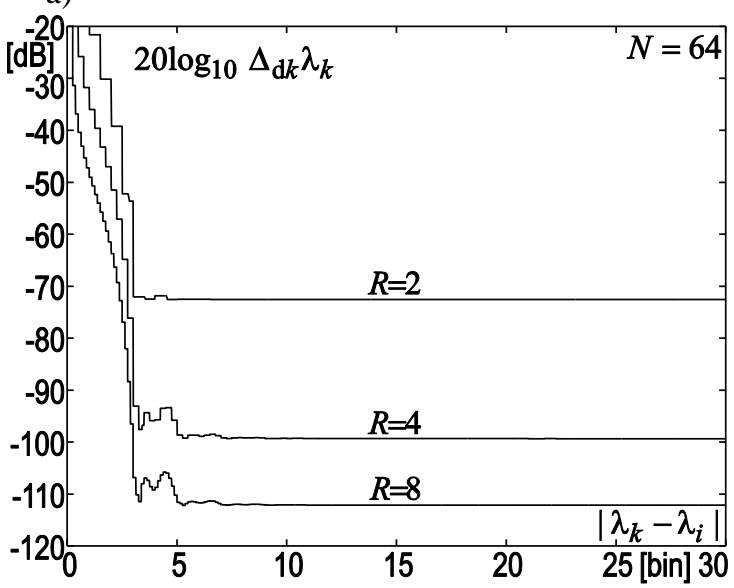

b)

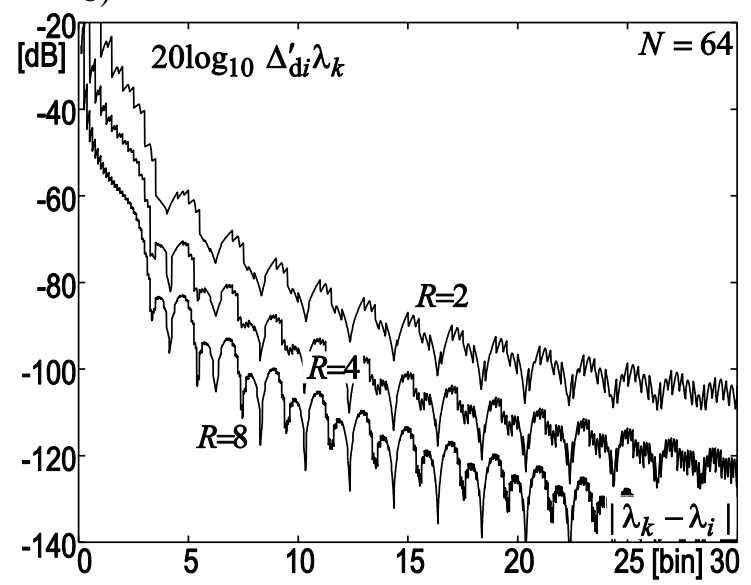

Fig. 2. Upper bounds of the component errors of the total error $\Delta_{\mathrm{d}} \lambda_{k}$ (25) in the estimation of $\lambda_{k}$ from (33)-(41) for a signal consisting of two complex oscillations, a triangular window $w_{n}$ in (9), $\eta_{1}=1 / 2$, and $\eta_{2}=1 / 6$ : a) $\Delta_{\mathrm{d} k} \lambda_{k}$, b) $\Delta_{\mathrm{d} i}^{\prime} \lambda_{k}$.

\section{Verification of the LIDFT systematic error equations using Monte Carlo simulations}

The upper bounds of the error components from matrix $\mathbf{D}$ defined by (33) are determined by (43). These bounds are obtained with some simplifying assumptions presented in previous sections, therefore the quality of these approximations must be verified. This verification was performed using simulations in which the samples of a signal consisting of two complex oscillations were processed in the LIDFT algorithm for a given parameter $R$ and the value of $\left|B_{i}\right| /\left|B_{k}\right|$. Each simulation was performed for $\lambda_{k}, \lambda_{i}$, $\arg B_{k}$, and $\arg B_{i}$ values that were randomly generated from across their full ranges. For this signal, the LIDFT algorithm with a triangular prototype window $w_{n}$ with $\eta_{1}=1 / 2$ and $\eta_{2}=1 / 6$ was applied to obtain the estimates of $\lambda_{k}$ and $\left|B_{k}\right|$, and the errors of these estimates were calculated. For each combination of the parameters $R$ and $\left|B_{i}\right| /\left|B_{k}\right|, 10^{7}$ simulations were performed, and the maximum values of the calculated errors are plotted against the distance $\left|\lambda_{k}-\lambda_{i}\right|$ between the components of the spectrum in comparison with the upper bounds determined from (33) and (43), as shown in Fig. 3.

a)

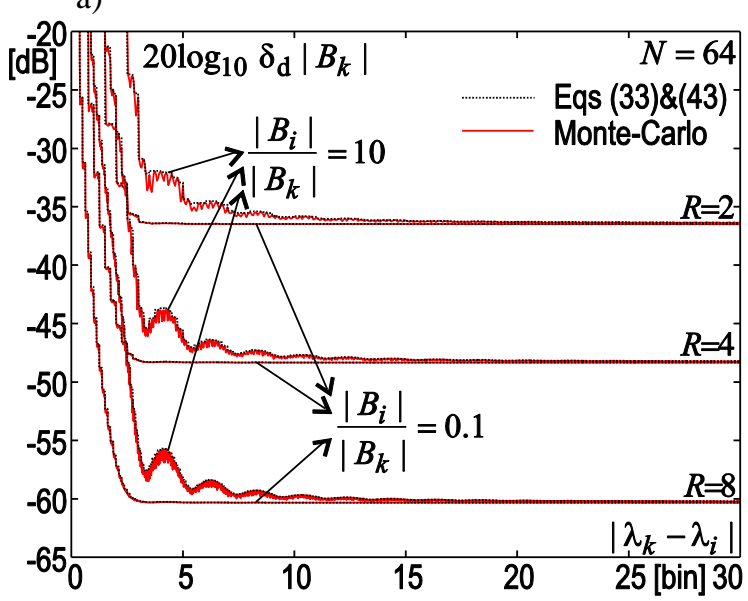

b)

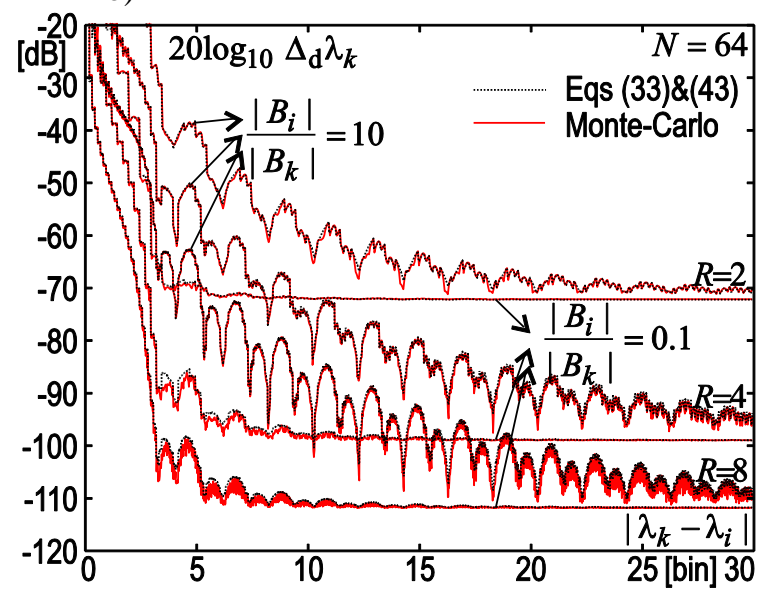

Fig. 3. Comparison of the upper bounds of the total systematic errors from (33) and (43)

of the LIDFT method (for the case of the triangular window $w_{n}$ in (9), $\eta_{1}=1 / 2$, and $\eta_{2}=1 / 6$ ) with Monte-Carlo simulation results (with $10^{7}$ simulations for each curve) for $R=2,4,8$ and $\left|B_{i}\right| /\left|B_{k}\right|=0.1,10$ : a) the error of the amplitude estimation, $\delta_{\mathrm{d}}\left|B_{k}\right|$; b) the error of the frequency estimation, $\Delta_{\mathrm{d}} \lambda_{k}$. 
The ranges of the plotted errors are limited to the values below $10 \%$ for $\delta_{\mathrm{d}}\left|B_{k}\right|$ (i.e., -20 $\mathrm{dB}$ ) and below 0.1 bin for $\Delta_{\mathrm{d}} \lambda_{k}$ (i.e., $-20 \mathrm{~dB}$ with respect to $1 \mathrm{bin}$ ). Over these ranges, the results presented here converge well to the upper bounds of the errors obtained in Section 4 and the maximum errors of the LIDFT method obtained in the simulations.

\section{Conclusions}

The main results of this paper are analytical formulas for the systematic errors of the LIDFT method and the verification of these formulas using Monte-Carlo simulations. The analysis presented here shows that for multifrequency signals consisting of many complex oscillations, the total systematic errors are the sum of the basic error components $\left(\delta_{\mathrm{d} k}\left|B_{k}\right|\right.$ for amplitude estimation and $\Delta_{\mathrm{d} k} \lambda_{k}$ for frequency estimation) and the components of each oscillation $\left(\delta_{\mathrm{d} i}\left|B_{k}\right|\right.$ and $\Delta_{\mathrm{d} i} \lambda_{k}$, which are proportional to the ratio $\left.\left|B_{i}\right| /\left|B_{k}\right|\right)$, as described by (22)-(27). Supplementing these relations with (32) yields analytical formulas for some particular cases. One of the basic cases is a signal consisting of two complex oscillations, analyzed in Sect. 4. The main results of this section are relations (35)-(41) and (43), which together determine each error component for the case of two complex oscillations (basic components $\delta_{\mathrm{d} k}\left|B_{k}\right|, \Delta_{\mathrm{d} k} \lambda_{k}$ and additional components $\delta_{\mathrm{d} i}^{\prime}\left|B_{k}\right|, \Delta_{\mathrm{d} i}^{\prime} \lambda_{k}$, which multiplied by the ratio $\left|B_{i}\right||| B_{k} \mid$ are the components $\delta_{\mathrm{d} i}\left|B_{k}\right|, \Delta_{\mathrm{d} i} \lambda_{k}$ ). The plots of these four components $\left(\delta_{\mathrm{d} k}\left|B_{k}\right|, \Delta_{\mathrm{d} k} \lambda_{k}, \delta_{\mathrm{d} i}^{\prime}\left|B_{k}\right|, \Delta_{\mathrm{d} i}^{\prime} \lambda_{k}\right)$ in Figs. 1 and 2 show that increasing the parameter $R$ decreases all of these error components and that the errors increase with small distances between components of the spectrum. The total estimation errors $\left(\delta_{\mathrm{d}}\left|B_{k}\right|\right.$ and $\left.\Delta_{\mathrm{d}} \lambda_{k}\right)$ for the case of two complex oscillations described by (33) are determined based on analytical solutions from Section 4 and verified using Monte-Carlo simulations with randomly generated frequencies and phases of complex oscillations (Fig. 3). Each curve was subjected to $10^{7}$ simulations, although far smaller numbers of simulations yielded similar results. These results demonstrate the accuracy of the analytical solutions presented in Section 4 over practical ranges of the errors from Figs. 1-3, i.e., below $-20 \mathrm{~dB}$. These results characterize the basic properties of the LIDFT method and its systematic errors, and they can be used to improve this method for the estimation of multifrequency signal parameters.

\section{Appendix A. Derivation of (22)-(24)}

From (21) and $M_{k k} \approx 1$ (due to the case of small systematic errors of phase $\arg B_{k}$ and modulus $\left.\left|B_{k}\right|\right)$ :

$$
\begin{aligned}
\delta_{\mathrm{d}}\left|B_{k}\right| & =\max _{\substack{\arg B_{i} \\
i \in S_{1}}}\left|\frac{\left|\hat{B}_{k}\right|-\left|B_{k}\right|}{\left|B_{k}\right|}\right|=\max _{\substack{\arg B_{i} \\
i \in S_{1}}}\left|\frac{\left|\hat{B}_{k}\right|}{\left|B_{k}\right|}-1\right|=\underset{\substack{\arg B_{i} \\
i \in S_{1}}}{\max }|| M_{k k}+\sum_{\substack{i \in S_{1} \\
i \neq k}} M_{k i} \frac{B_{i}}{B_{k}}|-1| \\
& =\left|M_{k k}-1\right|+\sum_{\substack{i \in S_{1} \\
i \neq k}} \frac{\left|B_{i}\right|}{\left|B_{k}\right|}\left|M_{k i}\right|=\delta_{\mathrm{d} k}\left|B_{k}\right|+\sum_{\substack{i \in S_{1} \\
i \neq k}} \delta_{\mathrm{d} i}\left|B_{k}\right|,
\end{aligned}
$$

where $\delta_{\mathrm{d} k}\left|B_{k}\right|$ and $\delta_{\mathrm{d} i}\left|B_{k}\right|$ are defined by (23) and (24).

\section{Appendix B. Derivation of (25)-(27)}

To obtain high accuracy, i.e., $\delta_{\mathrm{d}}\left|B_{k}\right|<<1$, the condition $\left|M_{k i}\right| \cdot\left|B_{i}\right| /\left|B_{k}\right|<<1$ must be fulfilled. Using this condition, (16), (21) and an expansion to a Maclaurin series with respect to $M_{k i} B_{i} / B_{k}$ : 


$$
\begin{aligned}
\Delta_{\mathrm{d}} \lambda_{k} & =\max _{\substack{\arg B_{i} \\
i \in S_{1}}}\left|\hat{\lambda}_{k}-\lambda_{k}\right|=\max _{\substack{\arg B_{i} \\
i \in S_{1}}} \frac{1}{R}\left|\operatorname{Re} \frac{\hat{C}_{k}}{\hat{B}_{k}}-\gamma_{k}\right|=\max _{\substack{\arg B_{i} \\
i \in S_{1}}} \frac{1}{R}\left|\operatorname{Re} \frac{\sum_{i \in S_{1}} N_{k i} B_{i}}{\sum_{i \in S_{1}} M_{k i} B_{i}}-\gamma_{k}\right| \\
& =\max _{\substack{\arg B_{i} \\
i \in S_{1}}} \frac{1}{R}\left|\operatorname{Re} \frac{N_{k k}+\sum_{i \in S_{1}, i \neq k} \frac{N_{k i}}{M_{k i}}\left(M_{k i} \frac{B_{i}}{B_{k}}\right)}{M_{k k}+\sum_{i \in S_{1}, i \neq k}\left(M_{k i} \frac{B_{i}}{B_{k}}\right)}-\gamma_{k}\right| \\
& \cong \max _{\substack{\arg B_{i} \\
i \in S_{1}}} \frac{1}{R}\left|\operatorname{Re} \frac{N_{k k}}{M_{k k}}-\gamma_{k}+\operatorname{Re} \sum_{i \in S_{1}, i \neq k} \frac{1}{M_{k k}}\left(N_{k i}-\frac{N_{k k}}{M_{k k}} M_{k i}\right)\right| \\
& =\frac{1}{R}\left|\operatorname{Re} \frac{N_{k k}}{M_{k k}}-\gamma_{k}\right|+\sum_{i \in S_{1}, i \neq k} \frac{\left|B_{i}\right|}{\left|B_{k}\right|} \cdot \frac{1}{R}\left|\frac{1}{M_{k k}}\left(N_{k i}-\frac{N_{k k}}{M_{k k}} M_{k i}\right)\right|,
\end{aligned}
$$

yielding (25) with:

$$
\begin{gathered}
\Delta_{\mathrm{d} k} \lambda_{k}=R^{-1}\left|\operatorname{Re} \frac{N_{k k}}{M_{k k}}-\gamma_{k}\right| \leq R^{-1}\left|\frac{N_{k k}}{M_{k k}}-\gamma_{k}\right|, \\
\Delta_{\mathrm{d} i} \lambda_{k}=\sum_{i \in S_{1}, i \neq k} \frac{\left|B_{i}\right|}{\left|B_{k}\right|} \cdot \Delta_{\mathrm{d} i}^{\prime} \lambda_{k}, \quad \Delta_{\mathrm{d} i}^{\prime} \lambda_{k}=R^{-1}\left|\frac{1}{M_{k k}}\left(N_{k i}-\frac{N_{k k}}{M_{k k}} M_{k i}\right)\right| .
\end{gathered}
$$

For small systematic errors $\delta_{\mathrm{d} k}\left|B_{k}\right|<<1$, i.e., $\left|M_{k k}-1\right|<<1$; thus, the following approximation can be made:

$$
M_{k k}^{-1}=\left[1+\left(M_{k k}-1\right)\right]^{-1}=1-\left(M_{k k}-1\right)+o\left(\left(M_{k k}-1\right)^{2}\right) \approx 1-\left(M_{k k}-1\right),
$$

which, when used in (B2)-(B3), using (23)-(24) and under the conditions:

$$
\delta_{\mathrm{d} k}\left|B_{k}\right|<<1, \quad \delta_{\mathrm{d} i}^{\prime}\left|B_{k}\right|<<1, \quad \Delta_{\mathrm{d} k} \lambda_{k}<<1, \quad \Delta_{\mathrm{d} i}^{\prime} \lambda_{k}<<1,
$$

yields (26)-(27):

$$
\begin{aligned}
& \Delta_{\mathrm{d} k} \lambda_{k} \cong R^{-1}\left|N_{k k}-\gamma_{k}-\left(N_{k k}-\gamma_{k}+\gamma_{k}\right)\left(M_{k k}-1\right)\right| \cong R^{-1}\left|\left(N_{k k}-\gamma_{k}\right)-\gamma_{k}\left(M_{k k}-1\right)\right| \\
& \Delta_{\mathrm{d} i}^{\prime} \lambda_{k}=R^{-1}\left|\frac{N_{k i}\left(M_{k k}-1\right)+N_{k i}-\gamma_{k} M_{k i}-\left(N_{k k}-\gamma_{k}\right) M_{k i}}{\left[1+\left(M_{k k}-1\right)\right]^{2}}\right| \cong R^{-1}\left|N_{k i}-\gamma_{k} M_{k i}\right| .
\end{aligned}
$$

\section{Appendix C. Derivation of (35)}

For the signal consisting of two complex oscillations $B_{k} e^{j 2 \pi n \lambda_{k} / N}$ and $B_{i} e^{j 2 \pi n \lambda_{i} / N}$ defined in the first paragraph of Section 4, (32) has the form:

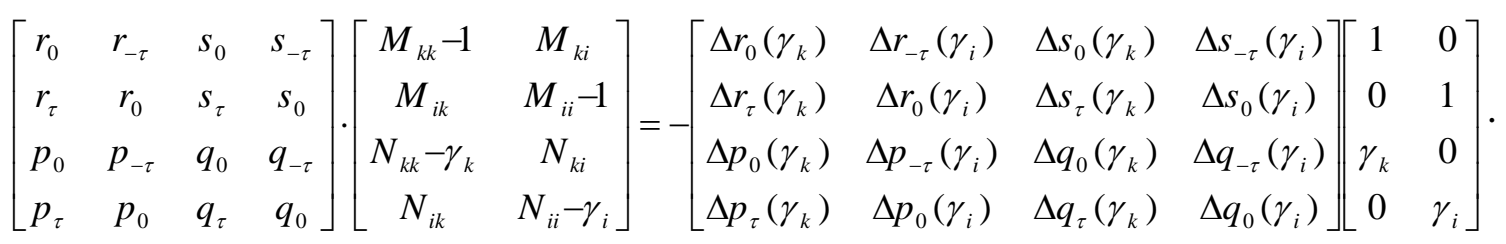

The property of an even $h_{n}$, taking into account (6) and (9), means that $g_{n}$ is odd. From this assumption and based on (19), function $H(\lambda)$ is even with respect to $\lambda$, and function $G(\lambda)$ is odd, and taking into account (18): 


$$
H_{-\tau}(-\gamma)=H_{\tau}(\gamma), \quad G_{-\tau}(-\gamma)=-G_{\tau}(\gamma) .
$$

Condition $h_{-N / 2}=0$ yields the condition $g_{-N / 2}=0$. From this and from the even and odd properties of $h_{n}$ and $g_{n}$, respectively, it follows that the coefficients $r_{m}, s_{m}, p_{m}$, and $q_{m}$ from (12)-(13) are real and fulfill the following:

$$
r_{-\tau}=r_{\tau}, s_{-\tau}=-s_{\tau}, p_{-\tau}=-p_{\tau}, q_{-\tau}=q_{\tau}, s_{0}=p_{0}=0 .
$$

Analogous conditions are also fulfilled for (29)-(30):

$$
\Delta r_{-\tau}=\Delta r_{\tau}, \Delta s_{-\tau}=-\Delta s_{\tau}, \Delta p_{-\tau}=-\Delta p_{\tau}, \Delta q_{-\tau}=\Delta q_{\tau}, \Delta s_{0}=\Delta p_{0}=0 .
$$

Equation $(\mathrm{C} 1)$, taking into account $(\mathrm{C} 3)-(\mathrm{C} 4)$, has the form:

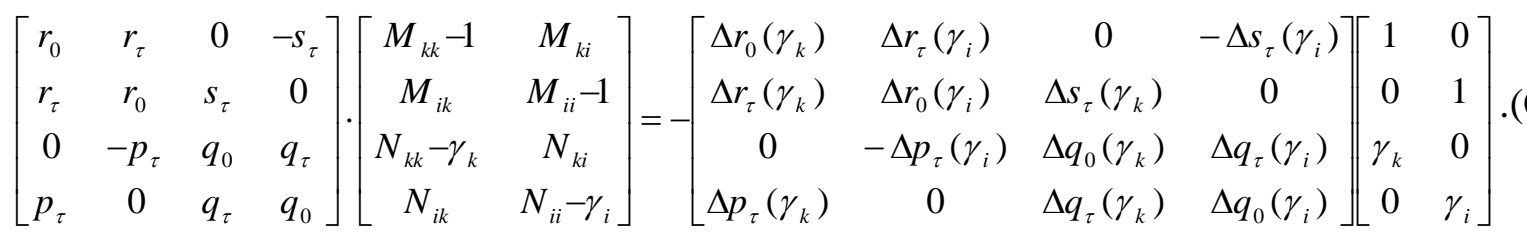

To shorten the notations of the next transformations, the 18 symbols $(a, b, \ldots)$ are introduced as follows:

$$
\begin{gathered}
a=r_{0}-r_{\tau}, b=-s_{\tau}, c=p_{\tau}, d=q_{0}+q_{\tau}, e=r_{0}+r_{\tau}, f=q_{0}-q_{\tau}, \\
u=\gamma_{k} \Delta s_{\tau}\left(\gamma_{k}\right), v=\Delta r_{0}\left(\gamma_{k}\right)-\Delta r_{\tau}\left(\gamma_{k}\right), w=\Delta r_{0}\left(\gamma_{k}\right)+\Delta r_{\tau}\left(\gamma_{k}\right), \\
u^{\prime}=\gamma_{i} \Delta s_{\tau}\left(\gamma_{i}\right), v^{\prime}=\Delta r_{0}\left(\gamma_{i}\right)-\Delta r_{\tau}\left(\gamma_{i}\right), w^{\prime}=\Delta r_{0}\left(\gamma_{i}\right)+\Delta r_{\tau}\left(\gamma_{i}\right), \\
x=\Delta p_{\tau}\left(\gamma_{k}\right), y=\gamma_{k}\left(\Delta q_{0}\left(\gamma_{k}\right)-\Delta q_{\tau}\left(\gamma_{k}\right)\right), z=\gamma_{k}\left(\Delta q_{0}\left(\gamma_{k}\right)+\Delta q_{\tau}\left(\gamma_{k}\right)\right), \\
x^{\prime}=\Delta p_{\tau}\left(\gamma_{i}\right), y^{\prime}=\gamma_{i}\left(\Delta q_{0}\left(\gamma_{i}\right)-\Delta q_{\tau}\left(\gamma_{i}\right)\right), z^{\prime}=\gamma_{i}\left(\Delta q_{0}\left(\gamma_{i}\right)+\Delta q_{\tau}\left(\gamma_{i}\right)\right) .
\end{gathered}
$$

After transformation (C5), by summing the appropriate columns and rows and taking into account (C6)-(C10):

$$
\begin{aligned}
& {\left[\begin{array}{cccc}
e & a & b & -b \\
e & -a & -b & -b \\
-c & c & d & f \\
c & c & d & -f
\end{array}\right] \cdot\left[\begin{array}{cc}
\left(M_{k k}-1\right)+M_{i k} & M_{k i}+\left(M_{i i}-1\right) \\
\left(M_{k k}-1\right)-M_{i k} & M_{k i}-\left(M_{i i}-1\right) \\
\left(N_{k k}-\gamma_{k}\right)+N_{i k} & N_{k i}+\left(N_{i i}-\gamma_{i}\right) \\
\left(N_{k k}-\gamma_{k}\right)-N_{i k} & N_{k i}-\left(N_{i i}-\gamma_{i}\right)
\end{array}\right]=-\left[\begin{array}{cc}
w+v & w^{\prime}-v^{\prime}-2 u^{\prime} \\
w-v+2 u & w^{\prime}+v^{\prime} \\
y+z & z^{\prime}-y^{\prime}-2 x^{\prime} \\
z-y+2 x & z^{\prime}+y^{\prime}
\end{array}\right],} \\
& {\left[\begin{array}{cccc}
e & 0 & 0 & -b \\
0 & a & b & 0 \\
0 & c & d & 0 \\
-c & 0 & 0 & f
\end{array}\right] \cdot\left[\begin{array}{cc}
\left(M_{k k}-1\right)+M_{i k} & M_{k i}+\left(M_{i i}-1\right) \\
\left(M_{k k}-1\right)-M_{i k} & M_{k i}-\left(M_{i i}-1\right) \\
\left(N_{k k}-\gamma_{k}\right)+N_{i k} & N_{k i}+\left(N_{i i}-\gamma_{i}\right) \\
\left(N_{k k}-\gamma_{k}\right)-N_{i k} & N_{k i}-\left(N_{i i}-\gamma_{i}\right)
\end{array}\right]=-\left[\begin{array}{cc}
w+u & w^{\prime}-u^{\prime} \\
v-u & -v^{\prime}-u^{\prime} \\
z+x & z^{\prime}-x^{\prime} \\
y-x & -y^{\prime}-x^{\prime}
\end{array}\right] .}
\end{aligned}
$$

Omitting elements equal to zero in (C12), two equations are obtained instead of (C5):

$$
\begin{gathered}
{\left[\begin{array}{ll}
a & b \\
c & d
\end{array}\right] \cdot\left[\begin{array}{cc}
\left(M_{k k}-1\right)-M_{i k} & M_{k i}-\left(M_{i i}-1\right) \\
\left(N_{k k}-\gamma_{k}\right)+N_{i k} & N_{k i}+\left(N_{i i}-\gamma_{i}\right)
\end{array}\right]=-\left[\begin{array}{cc}
v-u & -v^{\prime}-u^{\prime} \\
z+x & z^{\prime}-x^{\prime}
\end{array}\right],} \\
{\left[\begin{array}{cc}
e & -b \\
-c & f
\end{array}\right] \cdot\left[\begin{array}{cc}
\left(M_{k k}-1\right)+M_{i k} & M_{k i}+\left(M_{i i}-1\right) \\
\left(N_{k k}-\gamma_{k}\right)-N_{i k} & N_{k i}-\left(N_{i i}-\gamma_{i}\right)
\end{array}\right]=-\left[\begin{array}{cc}
w+u & w^{\prime}-u^{\prime} \\
y-x & -y^{\prime}-x^{\prime}
\end{array}\right],}
\end{gathered}
$$


and multiplying the left sides of these equations by the inverses of the appropriate matrices and summing by sides yields (35) and (36).

\section{References}

[1] Marple, S.L. (1987). Digital Spectral Analysis with Applications, Prentice-Hall.

[2] Kay, S.M. (1988). Modern Spectral Estimation: Theory and Application, Englewood Cliffs, Prentice-Hall.

[3] Scharf, L.L. (1991). Statistical Signal Processing: Detection, Estimation and Time Series Analysis, Addison-Wesley.

[4] Mitra, S.K., Kaiser, J.F. (1993). Handbook for Digital Signal Processing, Wiley.

[5] Szmajda, M., Górecki, K., Mroczka, J. (2010). Gabor transform, spwvd, gabor-wigner transform and wavelet transform - tools for power quality monitoring. Metrol. Meas. Syst., 17(3), 383-396.

[6] Mroczka, J., Szczuczyński, D. (2009). Inverse problems formulated in terms of first-kind Fredholm integral equations in indirect measurements. Metrol. Meas. Syst., 16(3), 333-357.

[7] Zygarlicki, J., Zygarlicka, M., Mroczka, J., Latawiec, K.J. (2010). A reduced Prony's method in powerquality analysis-parameters selection. IEEE Trans. Power Del., 25(2), 979-986.

[8] Zygarlicki, J., Mroczka, J. (2011). Short time algorithm of power waveforms fundamental harmonic estimation with use of prony's methods. Metrol. Meas. Syst., 18(3), 371-378.

[9] Zivanovic, M., Carlosena, A. (2001). Nonparametric Spectrum Interpolation Methods: A Comparative Study. IEEE Trans. Instrum. Meas., 50(5), 1127-1132.

[10] Zivanovic, M., Carlosena, A. (2002). Extending the limits of resolution for narrow-band harmonic and modal analysis: a non-parametric approach. Measurement Science and Technology, 13(12), 2082-2089.

[11] Aiello, M., Cataliotti, A., Nuccio, S. (2005). A Chirp-Z Transform-Based Synchronizer for Power System Measurements. IEEE Trans. Instrum. Meas., 54(3), 1025-1032.

[12] Sarkar, I., Fam, A.T. (2006). The interlaced chirp Z transform. Signal Processing, 86, 2221-2232.

[13] Duda, K., Borkowski, D., Bień, A. (2009). Computation of the network harmonic impedance with Chirp-Z transform. Metrol. Meas. Syst., 16(2), 299-312.

[14] Makur, A., Mitra, S.K. (2001). Warped Discrete-Fourier Transform: Theory and Applications. IEEE Transactions on Circuits and Systems - I: Fundamental Theory and Applications, 48(9), 1086-1093.

[15] Franz, S.F., Mitra, S.K., Doblinger, G. (2003). Frequency estimation using warped discrete Fourier transform. Signal Processing, 83, 1661-1671.

[16] Venkataramanan, R., Prabhu, K.M.M. (2006). Estimation of frequency offset using warped discreteFourier transform. Signal Processing, 86, 250-256.

[17] Rife, D.C., Vincent, G.A. (1970). Use of the Discrete Fourier Transform in the Measurement of Frequencies and Levels of Tones. Bell System Technical Journal, 49, 197-228.

[18] Kamm, G.N. (1978). Computer Fourier-transform techniques for precise spectrum measurements of oscillatory data with application to the de Haas-van Alphen effect. J. of App. Phys., 49(12), 5951-5970.

[19] Jain, V.K., Collins, W.L., Davis, D.C. (1979). High-Accuracy Analog Measurements via Interpolated FFT. IEEE Trans. Instrum. Meas., 28(2), 113-121.

[20] Grandke, T. (1983). Interpolation Algorithms for Discrete Fourier Transforms of Weighted Signals. IEEE Transactions on Instrumentation and Measurement, 32, 350-355.

[21] Andria, G., Savino, M., Trotta, A. (1989). Windows and Interpolation Algorithms to Improve Electrical Measurement Accuracy. IEEE Trans. Instrum. Meas., 38(4), 856-863.

[22] Offelli, C., Petri, D. (1990). Interpolation Techniques for Real-Time Multifrequency waveform analysis. IEEE Trans. Instrum. Meas., 39(1), 106-111.

[23] Schoukens, J., Pintelon, R., Van Hamme, H. (1992). The Interpolated Fast Fourier Transform: A Comparative Study. IEEE Trans. Instrum. Meas., 41(2), 226-232. 
[24] Quinn, B.G. (1994). Estimating Frequency by Interpolation Using Fourier Coefficients. IEEE Transactions on Signal Processing, 42(5), 1264-1268.

[25] Quinn, B.G. (1997). Estimating of Frequency, Amplitude, and Phase from the DFT of a Time Series. IEEE Transactions on Signal Processing, 45(3), 814-817.

[26] Macleod, M.D. (1998). Fast Nearly ML Estimation of the Parameters of Real or Complex Single Tones or Resolved Multiple Tones. IEEE Transactions on Signal Processing, 46(1), 141-148.

[27] Sedlacek, M., Titera, M. (1998). Interpolations in frequency and time domains used in FFT spectrum analysis. Measurement, 23, 185-193.

[28] Santamaria, I., Pantaleon, C., Ibanez, J. (2000). A Comparative Study of High-Accuracy Frequency Estimation Methods. Mechanical Systems and Signal Processing, 14(5), 819-834.

[29] Borkowski, J. (2000). LIDFT - the DFT linear interpolation method. IEEE Trans. Instrum. Meas., 49(4), 741-745.

[30] Borkowski, J., Mroczka, J. (2000). Application of the discrete Fourier transform linear interpolation method in the measurement of volume scattering function at small angle. Optical Eng., 39(6), 1576-1586.

[31] Borkowski, J., Mroczka, J. (2002). Metrological analysis of the LIDFT method. IEEE Trans. Instrum. Meas., 51(1), 67-71.

[32] Agrež, D. (2002). Weighted Multipoint Interpolated DFT to Improve Amplitude Estimation of Multifrequency Signal. IEEE Transactions on Instrumentation and Measurement, 51(2), 287-292.

[33] Liguori, C., Paolillo, A. (2007). IFFTC-Based Procedure for Hidden Tone Detection. IEEE Trans. Instrum. Meas., 56(1), 133-139.

[34] Belega, D., Dallet, D. (2008). Frequency estimation via weighted multipoint interpolated DFT. IET Science, Measurement and Technology, 2(1), 1-8.

[35] Chen, K.F., Li, Y.F. (2008). Combining the Hanning windowed interpolated FFT in both directions. Computer Physics Communications, 178, 924-928.

[36] Li, Y.F., Chen, K.F. (2008). Eliminating the picket fence effect of the fast Fourier transform. Computer Physics Communications, 178, 486-491.

[37] Belega, D., Dallet, D. (2009). Multifrequency signal analysis by Interpolated DFT method with maximum sidelobe decay windows. Measurement, 42, 420-426.

[38] Yang, X.Z., Li, H.Y., Chen, K.F. (2009). Optimally averaging the interpolated fast Fourier transform in both directions. IET Science, Measurement and Technology, 3(2), 137-147.

[39] Chen, K.F., Jiang, J.T., Crowsen, S. (2009). Against the long-range spectral leakage of the cosine window family. Computer Physics Communications, 180, 904-911.

[40] Chen, K.F., Mei, S.L. (2010). Composite Interpolated Fast Fourier Transform With the Hanning Window. IEEE Trans. Instrum. Meas., 59(6), 1571-1579.

[41] Borkowski, J., Mroczka, J. (2010). LIDFT method with classic data windows and zero padding in multifrequency signal analysis. Measurement, 43, 1595-1602.

[42] Borkowski, J. (2011). Methods of spectrum interpolation and the LIDFT method for estimation of multifrequency signal parameters. Publishing House of Wrocław University of Technology, Wrocław, Poland. (in Polish)

[43] Duda, K. (2011). DFT Interpolation Algorithm for Kaiser-Bessel and Dolph-Chebyshev Windows. IEEE Trans. Instrum. Meas., 60(3), 784-790.

[44] Belega, D., Dallet, D., Petri, D. (2012). Statistical description of the sine-wave frequency estimator provided by the interpolated DFT method. Measurement, 45(1), 109-117

[45] Borkowski, J. (2011). Minimization of maximum errors in universal approximation of the unit circle by a polygon. Metrol. Meas. Syst., 18(3), 391-402.

[46] Borkowski, J. (2011). Optimization of the unit circle approximation by a polygon. Electrical Review, 87(7), 95-99. 